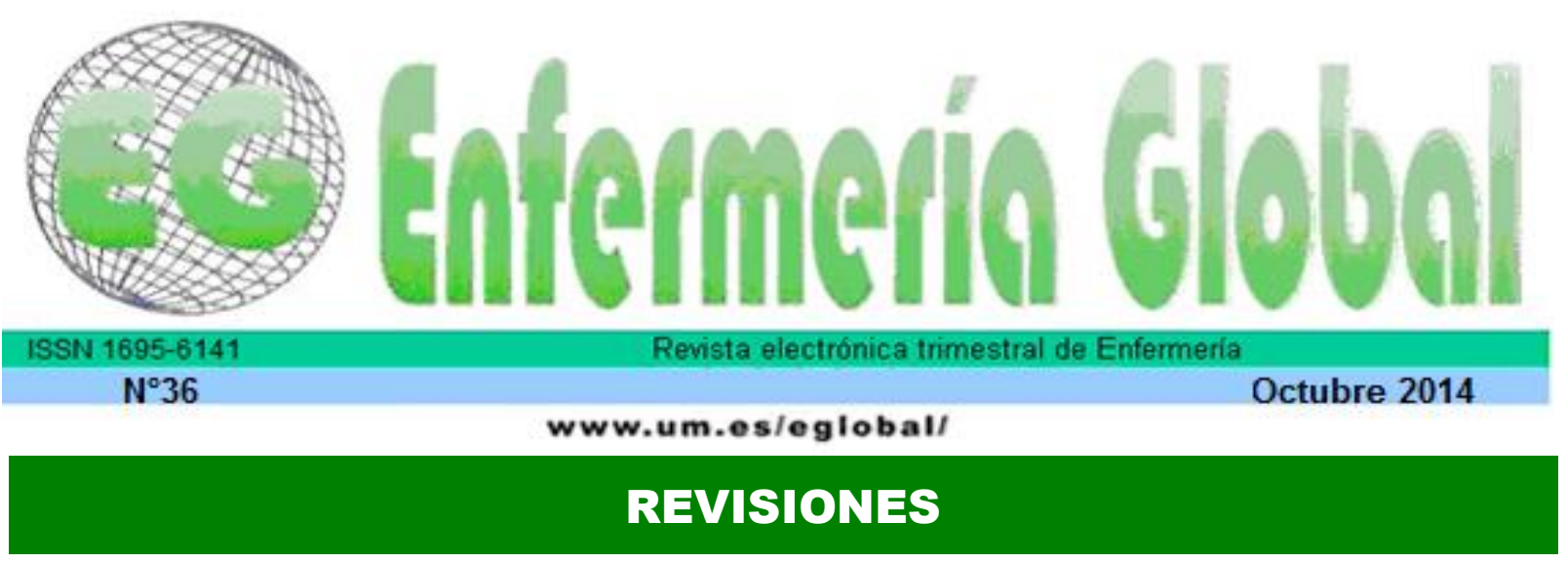

\title{
Calidad de servicio: satisfacción usuaria desde la perspectiva de enfermería
}

Quality of service: user satisfaction from a nursing perspective

\author{
*Gallardo Ferrada, Amanda **Reynaldos Grandón, Katiuska \\ *Enfermera. Magíster en Enfermería Mención Gestión. Facultad de Medicina, Pontificia Universidad \\ Católica de Chile. E-mail: aigallar@uc.cl **Enfermera Matrona. Ingeniero Comercial. MBA. Phd \\ Ciencias Empresariales. Facultad de Enfermería. Universidad Andrés Bello.Chile. \\ Palabras clave: Administración; satisfacción usuaria; calidad de servicio.
}

Keywords: Administration; user satisfaction; service quality

\section{RESUMEN}

Uno de los aspectos relevantes y que en muchos casos determina la competitividad y permanencia en el mercado de las instituciones es la calidad de servicio. Frente a esto surge la necesidad de conocer cómo el usuario percibe y forma expectativas. Dentro de las empresas que aspiran a la calidad en su atención se encuentran las instituciones de salud siendo relevante identificar aspectos que influyen en la calidad de servicio.

Objetivo: Analizar los estudios realizados en el área de salud con enfoque en percepción y expectativas de servicio.

Diseño y método: Para esta revisión se realizó una búsqueda a través de las bases de datos: PubMed, Trip Database, Cochrane, además de Google Scholar y SciELO, con el propósito de analizar estudios relacionados con calidad de servicio. Los límites comprendieron: textos completos, idioma español y/o inglés, de los últimos 10 años y acceso gratuito. Se seleccionaron 17 artículos para la revisión.

Resultados: Todos los artículos comprenden la evaluación de la percepción y la calidad de servicio. De ellos 15 hacen referencia a servicios de salud.

Conclusiones: La calidad de servicio es percibida de diferentes maneras. Existen múltiples factores que determinan la percepción de calidad de servicio uno de ellos es el estado de salud de los usuarios.

\section{ABSTRACT}

One of the important aspects that in many cases determine the competitiveness and permanence in the market of institutions is the quality of service. In addition, there is a need to understand how the user perceives and forms expectations. Among those that aspire to identify what it is that influences the quality of care are health institutions. 
Objective: Analyze studies performed in the health field with a focus on the perception and expectation of service.

Design and method: Investigation of the following databases was performed for this review: PubMed, Trip Database, Cochrane, as well as Google Scholar and SciELO, in order to analyze studies related to the quality of service. The limits included: free access of complete texts, in Spanish and/or English, from the last 10 years. Seventeen articles were selected for review.

Results: All articles include the evaluation of perception and the quality of service. Fifteen of those articles refer to health services.

Conclusions: The quality of service is perceived in different ways. There are multiple factors that determine the perception of the quality of service, one of which is the user's state of health. Keywords: Administration, user satisfaction, quality of service.

\section{INTRODUCCIÓN}

La calidad de servicio se define, según el DRAE, como "Propiedad o conjunto de propiedades inherentes a algo, que permiten juzgar su valor" (1). Montgomery la define como "el grado en que los productos cumplen con las exigencias de la gente que los utiliza" (2)

Los servicios de salud, como toda empresa, deben enfrentar diversos desafíos, presiones, amenazas, para lograr mantener y desarrollar calidad en los servicios otorgados, deben saber cómo adecuar y adaptar las principales variables empresariales (tareas, tecnologías, estructura organizacional, personas y ambiente externo) en diferentes situaciones como: el surgimiento de nuevas tecnologías, la globalización, los cambios políticos, y los cambios en el conocimiento ${ }^{(3,4)}$.

\section{Calidad de servicio y satisfacción usuaria}

Estos factores explican el entorno competitivo en el que están inmersas las empresas, y por cierto también nuestras organizaciones de salud las cuales luchan por tener una adecuada coordinación de procesos como también la generación de condiciones que hagan que exista una diferencia con el resto de los competidores en el área de salud y que a su vez entregue valor a los servicios otorgados ${ }^{(5)}$.

Existen organizaciones de enfermeras que en búsqueda de características distintivas en el mercado enuncian como prioridad de investigación temas relacionados con la calidad de la atención y la satisfacción de sus clientes, por ejemplo: la American Association of Critical-care Nurses (AACN) publicó este tema como primera prioridad: "empleo efectivo y apropiado de la tecnología para conseguir la valoración, el tratamiento y los resultados óptimos del paciente" (6). La American Organization of Nurse Executives (AONE) estableció como prioridad de investigación para el año 2000: 1) estudios sobre el personal, 2) defensa del cuidado del paciente y 3) tecnología ${ }^{(7)}$. La Agency for Healhcare Research and Quality (AHRQ) es una agencia que financia la investigación del cuidado sanitario y cuyo propósito es mejorar la calidad, adecuación y efectividad de los servicios sanitarios, promueve las mejoras de la práctica clínica, organización, financiación y prestación de servicios sanitarios ${ }^{(8)}$.

Al modificarse la naturaleza y distribución del mercado también lo hace el cliente. El cliente ahora está más informado, es más selectivo, tiene mayor capacidad de elección, es más segmentado, tiene mayor nivel de vida, etc. Por esto se requiere monitorizar el nivel de satisfacción de los clientes hacia los servicios y productos, así 
el reto de la institución es hacia la innovación constante, con calidad del personal y orientación al cliente. Para lograr este enfoque hacia el cliente se debe empezar a identificar qué factores inciden sobre la satisfacción del usuario ${ }^{(9)}$. Estos factores en muchas ocasiones no se evidencian de manera consciente, sin embargo, pueden ser detectados y recogidos con el fin de poder entregar servicios que aspiren a la satisfacción de las necesidades de losv clientes.

\section{Evaluación de la calidad de servicio}

Existen diversas maneras de evaluar la calidad de servicio, siendo este punto una práctica relativamente reciente en nuestro país. En 1985 Parasuraman, Berry y Zeithaml, desarrollaron una herramienta para medir la calidad de servicio denominada SERVQUAL (Service Quality), enfocándose en la medición de la brecha existente entre percepciones y expectativas de los usuarios respecto del servicio otorgado. Cuando hablamos de percepción nos referimos a cómo el cliente estima que la institución cumple al proporcionar los servicios. Expectativas se define como lo que espera que sea el servicio que entrega la organización ${ }^{(10)}$.

Estos factores que influyen en la satisfacción usuaria son aspectos cada vez más estudiados en nuestro país, encontrando algunas investigaciones, que serán analizadas más adelante, realizadas en organizaciones de salud, principalmente hospitalarias en las que se evalúa la perspectiva del cliente con el fin de conseguir información para mejorar atención y procesos.

\section{Satisfacción usuaria en Chile}

Esta problemática es relevante en nuestro país, el Ministerio de Salud (MINSAL) lo enuncia dentro de su Misión en donde busca "....acoger oportunamente las necesidades de las personas, familias y comunidades, con la obligación de rendir cuentas a la ciudadanía y promover la participación de las mismas en el ejercicio de sus derechos y sus deberes" (11). Al MINSAL le corresponde formular y fijar las políticas de salud. En consecuencia tiene, entre otras funciones, la elaboración de los objetivos sanitarios para la década. Dentro de los objetivos sanitarios destacamos el número IV que consiste en "Proveer servicios acorde a las expectativas de la población", planteándose objetivos como la satisfacción de las expectativas legítimas de la población, buscando aumentar el grado de satisfacción de la población con su sistema de salud ${ }^{(12)}$. Desde esta perspectiva, y según los resultados obtenidos, las nuevas metas en materia de satisfacción usuaria deberán orientarse a redefinir el vínculo entre las personas y el sector, promover el ejercicio pleno de los derechos y las responsabilidades de las personas en salud y relevar las dimensiones significativas en materia de satisfacción usuaria. Además, el desafío será incorporar las dimensiones de satisfacción usuaria a la formación académica de los profesionales médicos, paramédicos y técnicos, pues la orientación hacia el usuario es una preocupación que debe instalarse tempranamente entre quienes formarán equipos de atención ${ }^{(13)}$

Por lo expuesto anteriormente y por ser un tema en desarrollo a nivel país y sobre todo en el área de salud surge la necesidad de analizar estas dimensiones. El presente artículo expone: la percepción y satisfacción del usuario, la calidad de servicio de salud y finalmente la discusión y conclusión del tema expuesto. 


\section{METODOLOGÍA}

Se realizó una revisión integradora, permitiendo la inclusión simultánea de investigaciones cuantitativas (experimental y no experimental) y cualitativas con el fin de comprender mejor el fenómeno de interés. Para esta revisión se realizó una búsqueda a través de las bases de datos: Pubmed, Tripdatabase, Cochrane, además de Google Scholar y Scielo, de acceso gratuito, con el propósito de analizar estudios relacionados con la calidad de servicio, percepción de usuarios y expectativas que condicionan su satisfacción frente a los servicios otorgados, con el objeto de tener una visión general de cómo el cliente percibe y genera expectativas de un determinado servicio. Se utilizaron las siguientes palabras claves en español e inglés respectivamente: percepción, administración, satisfacción usuaria, calidad de servicio, administration, user satisfaction, service quality. Se consideró la disponibilidad gratuita del texto completo, idioma español y/o inglés. Se excluyeron aquellos trabajos de más de 10 años de antigüedad y aquellos en que se encontraba disponible solamente el resumen y no pudo ser considerado el texto completo al momento de revisar las bases de datos. Para la selección de los artículos se consideró la opinión de expertos en el área, para dirigir y acotar la búsqueda del tema, como así también los límites de la investigación. Finalmente, se analizaron 17 artículos de investigación de los cuales 11 utilizan metodología cuantitativa, 3 metodología cualitativa, 1 utiliza método mixto (cuali-cuantitativa) y 2 corresponden a revisiones de literatura. De la totalidad de artículos 10 son textos en español y 7 son en inglés. Para esto se plantea la siguiente pregunta de investigación, ¿cómo condiciona la percepción y las expectativas del usuario la satisfacción en relación a los servicios de salud otorgados?

\section{RESULTADOS}

\section{Calidad de servicio}

La calidad de servicio debe cumplir con ciertas dimensiones como son: la dimensión técnica referida a que el prestador cuente con conocimientos, habilidades para la realización de procedimientos clínicos adecuados y oportunos de conformidad con las necesidades del cliente, la dimensión interpersonal referida a la interacción entre prestador y cliente en la actividad de atención, respeto y cordialidad mutua, por último, la dimensión de infraestructura que son las características del lugar como la limpieza, iluminación y ventilación del ambiente ${ }^{(14)}$

Velandia et al. ${ }^{(15)}$ señala que la oportunidad y la calidad de los servicios de atención fueron bien evaluadas por parte de la población colombiana. Así mismo, se identificaron algunos factores que determinan las variaciones en las evaluaciones de la oportunidad y de la calidad, lo mismo que en las razones para no utilizar los servicios de atención médica. En relación a las razones de no uso de los servicios, se encontró que 64,7 \% de la población que no utilizó los servicios de atención médica, mencionó "razones" que se asocian con problemas de calidad. Entre las más importantes, se encontraron las relacionadas con la accesibilidad: larga distancia hasta el oferente, falta de dinero y falta de credibilidad en los agentes de salud. Se identificó un conjunto de factores o variables que se asociaron tanto con las variaciones en la oportunidad como con las variaciones en la calidad (satisfacción), entre los que cabe destacar la afiliación a la seguridad social. Otros factores, como el plan de salud utilizado para acceder a la atención médica, se asociaron únicamente con la oportunidad, y el que tiene que ver con la entidad de afiliación, se asoció sólo con la calidad o satisfacción. Sin embargo, la calidad de los servicios en general fue 
bien evaluada. Además el tiempo de atención, el trato del personal, la gestión de capacidades y compromisos orientados al usuario, gestión de información y coordinación entre los servicios de información, reclamos y gestión de los datos del usuario fueron aspectos influyentes en la calidad global percibida por el cliente ${ }^{(16)}$.

Otro aspecto a considerar en la evaluación de la calidad de servicio es el estado de salud percibida del cliente. Los usuarios con mal estado de salud mental tienen sentencias menos positivas de los aspectos que están relacionados con la accesibilidad a los servicios y los aspectos interpersonales de la atención. El mal estado de salud física, en cambio, predice sentencias menos positivas de aspectos tales como la limpieza del edificio, la confidencialidad de la consulta con el médico de cabecera, entre otros, siendo todos estos aspectos estadísticamente significativos ${ }^{(17)}$. Una revisión sistemática realizada al respecto por Patterson, et al. ${ }^{(18)}$ en Reino Unido, evidencia que en la literatura de salud la participación de los empleados en grupos de mejora continua de la calidad, obtienen mejores resultados de salud en sus usuarios. Desde otra perspectiva un estudio realizado en EEUU evidencia que en los hospitales que tienen un mayor número de personal de enfermería puede estar asociado con una mejor experiencia para los pacientes ${ }^{(19)}$.

En relación a la contribución de enfermería a la calidad de servicio, los datos por si solos no proporcionan toda una imagen, es necesario evaluar el lugar de trabajo, el trabajo en equipo, sistemas y liderazgo. Además los incentivos para el cambio de comportamiento tanto a nivel individual como a nivel sistémico son relevantes para influir finalmente en la calidad de atención de los usuarios. Una enfermería de calidad debe basarse en un cuidado: centrado en la persona, identificando sus valores, necesidades y expectativas con respecto a su propia salud y asistencia social; comunicar y facilitar la información pertinente, que permite compartir la toma de decisiones, elección informada, y permitir la participación en la evaluación de la atención. Por otro lado, el cuidado debe ser efectivo, es decir, un cuidado seguro y basado en la evidencia en relación a los tratamientos previstos y el contexto en el que la atención se lleva a cabo y, finalmente, debe considerar los sistemas de atención en lo relativo al contexto en que se presta la asistencia (por ejemplo, sistemas de seguridad, recursos humanos, la continuidad de la atención), incluyendo las estructuras, procesos y patrones de comportamiento que permiten a las enfermeras centrarse en la persona, la atención segura y efectiva ${ }^{(20)}$.

Fitzpatrick citado en Caminal (21) refiere que "Existen tres razones por las que se debería considerar la satisfacción como una medida importante de resultado del proceso asistencial. Primero, hay trabajos que demuestran que la satisfacción es un buen predictor del cumplimiento del tratamiento por parte de los pacientes y de la adhesión a la consulta y al proveedor de servicios. En segundo lugar, la satisfacción es un instrumento útil para evaluar las consultas y los modelos de comunicación, por ejemplo, el éxito en informar o involucrar a los pacientes en la toma de decisiones sobre el tipo de atención. Y, en tercer lugar, la opinión del paciente puede utilizarse sistemáticamente para mejorar la organización de los servicios a proveer". La población debe ser partícipe de la mejora de la calidad de los servicios otorgados, ya que otorgan información esencial para conocer el funcionamiento de las instituciones desde la perspectiva del cliente y constituye un elemento de mejora de la calidad de la atención. 


\section{Percepción y Satisfacción usuaria}

Llanos et al. compara las escalas de Likert y Vigesimal para la evaluación de satisfacción de pacientes atendidos en un hospital. Los resultados obtenidos en satisfacción de la atención difieren según la escala usada para su medición. La elección de una determinada escala debe considerar los objetivos de la investigación, por lo que su importancia radica en lograr una adecuada medición de la calidad del servicio, que permita la toma de decisiones, con la finalidad de mejorar la calidad de este $^{(22)}$.

Salvador ${ }^{(23)}$ encontró que el cliente percibe de manera positiva la existencia de características estructurales como la eficacia y la tangibilidad, sin embargo, el cliente valora más el trato y las competencias de los profesionales. Existen ciertos aspectos percibidos como: largos tiempos de espera, falta de calidez en el trato y deficiencias en el confort, que influyen en el nivel de satisfacción de los usuarios (24). Riveros, et al. ${ }^{(25)}$ refiere que la naturaleza interactiva de los servicios de salud y su simultánea producción y consumo elevan la necesidad de examinar las percepciones de los implicados en el encuentro, tanto de profesionales como de usuarios, evidenciando que en relación a la variable compromiso profesional y ético, se valoró con percepciones inferiores por parte de los clientes. En relación a la variable preocupación del hospital, y al compromiso de los funcionarios por brindar calidad de atención, la percepción más positiva fue de los usuarios. Las opiniones más exigentes de los funcionarios son una autocrítica a sus responsabilidades que puede favorecer la aceptación de una aplicación efectiva de marketing avanzado en el establecimiento. Los funcionarios se mostraron más exigentes que los usuarios siguiendo una tendencia de autocrítica ${ }^{(25)}$.

Un estudio realizado a indígenas americanos mayores de 50 años referentes a sus percepciones con el proveedor de servicios de salud evidenció discordancia significativa entre las nueve formas de interacción entre paciente y prestador de salud. La discordancia refleja una tendencia en que los proveedores fueron más críticos de sus propias conductas que los pacientes. Estos hallazgos sugieren que los proveedores de servicio a menudo crean una mayor satisfacción de lo que creen (26). Desde la perspectiva del cliente Anden et al. ${ }^{(27)}$ evidenció que el resultado de acudir a una consulta médica es el de la cura o alivio de los síntomas, la compresión, la confirmación, tranquilidad, el cambio en la autopercepción y la satisfacción de sus necesidades.

Camgöz-Akdağ \& Zineldin ${ }^{(28)}$ sostienen que la satisfacción del cliente es una satisfacción acumulada que se construye sumando cinco factores "modelo $5 \mathrm{Qs}$ " que abarca técnicas, cualidades funcionales, la interacción, la infraestructura y el ambiente y servicios. De ellos, los tres factores de mayor preocupación para las personas que reciben tratamiento en hospitales en Turquía son: la calidad de la infraestructura; la calidad del ambiente la atmósfera y la calidad técnica. Además, existen deficiencias similares detectadas por los pacientes hospitalizados en Turquía, Jordania y Egipto las cuales fueron las habilidades y amabilidad de los médicos, la cortesía de las enfermeras y capacidad de respuesta a sus necesidades.

Riveros \& Berné $\left({ }^{(29)}\right.$ refiere que la satisfacción usuaria se logrará más fácilmente si se genera una cultura organizacional orientada al cliente y se apliquen políticas adecuadas de gestión y calidad de servicio utilizando tanto el marketing tradicional como los nuevos enfoques de orientación al mercado o al usuario y el marketing de 
relaciones. La consideración de estos nuevos enfoques de marketing como factores importantes en el logro de la satisfacción de las necesidades de los usuarios, incorpora al ámbito público la necesidad de un mayor y mutuo intercambio entre funcionarios y usuarios, lo que ayudaría a cambiar la mayor orientación al producto, que inicialmente comentábamos, por una mayor orientación al cliente o usuario. Estos aspectos de aplicación del marketing deberían integrarse efectivamente a los procesos de dirección de estos establecimientos hospitalarios (29). Riveros et al. ${ }^{(30)}$ estudia la relación entre la orientación al usuario y satisfacción con el servicio, con el objeto de centrarse en confirmar aquellos factores o componentes principales, que tanto en aspectos de orientación al usuario como en distintos elementos del servicio prestado, ejercen un mayor impacto sobre el nivel de satisfacción de los usuarios y pacientes de un establecimiento hospitalario. Esto permitirá construir una base para la puesta en marcha de mejoras en dichos centros asistenciales, con la consecuente mayor satisfacción de sus clientes.

\section{DISCUSIÓN}

Los temas revisados aportan información relevante de cómo los usuarios perciben en salud y qué aspectos se tornan más relevantes en determinadas situaciones. Los usuarios presentan altos niveles de satisfacción, sin embargo, de manera paralela se encuentran las quejas e insatisfacciones.

Fue importante evidenciar que los resultados en la satisfacción variarán de acuerdo al instrumento de recolección de datos seleccionado para llevar a cabo el estudio, de ahí la importancia en la experticia y formación de las personas que incurran en esta área con el fin de poder alinear correctamente la metodología con los objetivos que se persigan para llevar a cabo a nivel operacional eficazmente y eficientemente la investigación.

Se destaca también que las opiniones de la satisfacción con respecto a la calidad de atención tendrán mayor validez si se tiene en consideración el estado de salud del cliente que debe evaluarse de forma paralela.

En la valoración de la calidad del servicio influyen factores tangibles, sin embargo, el trato, las competencias profesionales y la orientación de la organización hacia el usuario, son factores que se repiten constantemente dentro de los resultados de las investigaciones, siendo este aspecto crucial a la hora de prestar atención sanitaria. El modelo 5Qs apunta a que debe existir una armonización entre distintas dimensiones (técnicas, cualidades funcionales, interacciones, infraestructura, calidad del ambiente y atmósfera y la calidad técnica) con el objeto de lograr una mayor satisfacción usuaria con el consiguiente mejoramiento de la calidad de atención.

En base al objetivo de esta revisión, el conocer cómo percibe un usuario y qué desencadena su nivel de satisfacción puede contribuir a rediseñar de manera creativa los procesos de gestión de calidad y orientación futura de sus estrategias. Esto reafirma que los hospitales pueden y deben ofrecer al mismo tiempo calidad de atención clínica y generar una buena experiencia para el usuario.

Desde esta perspectiva, la participación de los empleados de los servicios de salud en grupos de mejora continua se transforma en una estrategia que finalmente mejorará los resultados en lo que respecta a la calidad de la atención otorgada a los clientes. Sin embargo, esto es un fenómeno social que apunta a múltiples causas. Existen 
razones de no uso de los servicios de salud que se relacionan con: la previsión, los problemas geográficos que limitan el acceso y la falta de dinero de las familias. Para influir en estos aspectos se requiere de enfoques políticos y económicos que trabajen orientados en los determinantes sociales de la salud.

\section{CONCLUSIÓN}

La identificación de expectativas y percepciones de los usuarios en base a la calidad de servicio es esencial en los sistemas de salud, el MINSAL plantea esta problemática como un desafío destacando la necesidad de la incorporación de estos temas en la formación de los profesionales de salud.

Según lo expuesto en los resultados, existen estudios que revelan que un mayor número de enfermeras puede asociarse a una mejor experiencia por parte de los usuarios. Con esto se corrobora que la dimensión ontológica de nuestra disciplina es el cuidado, el cual debe ser centrado en el paciente, con un enfoque biopsicosocial apuntando hacia la integralidad en un contexto de respeto a sus valores e identificando sus necesidades y considerando las expectativas del usuario, todo esto con la finalidad de otorgar un cuidado de calidad. Es fundamental en la actual gestión integral de los servicios de salud, conocer a los clientes mediante información cuali y cuantitativa. La medición de estos aspectos se torna imprescindible debido a que si tenemos una población partícipe, que opina respecto a los servicios otorgados, tendremos una retroalimentación constante de aquellos puntos perfectibles en la práctica, mejorando los procesos y ayudando a que el trabajo en equipo tenga orientación al usuario como herramienta de mejora de la calidad.

\section{REFERENCIAS BIBLIOGRAFICAS}

1. "Calidad". En el Diccionario de la Real Academia de la Lengua Española [Internet]. s.f. Acceso 06 de May 2012. Hallado en: http://buscon.rae.es/drael/SrvltConsulta?LEMA=calidad

2. Montgomery DC. Introduction to statistical Quality Control. New York: Eds. John wiley \& Sons; 1985

3. Chiavenato I. Introducción a la teoría general administrativa. 3rd ed. México: Eds. McGraw. Hill; 2005.

4. d'Empaire G. Calidad de atención médica y principios éticos. Acta bioeth [Revista en Internet]. 2010 Nov. Acceso el 07 Mayo 2012; 16(2): 124-132. Hallado en: http://www.scielo.cl/scielo.php?script=sci_arttext\&pid=S1726569X2010000200004\&lng=es. doi: 10.4067/S1726-569X2010000200004.

5. Chiavenato, I. Administración de los recursos humanos. 5th ed. Colombia: Eds. McGraw. Hill; 2000.

6. Byers JF. Research Work Groups Announce Research Priorities for the New Millennium [Internet]. S.f. Acceso el 02 de Mayo de 2011, del sitio web de American Association of Critical Care Nurses. Hallado en: http://www.aacn.org/wd/practice/content/research/researchpriority.pcms?menu=practice

7. The American Organization of Nurse Executive. AONE 2009 Education and Research Priorities [Internet]. 2009. Acceso el 02 de Mayo 2011. Hallado en: http://www.aone.org/aone/edandcareer/priorities.html

8. Agency for Healthcare Research and Quality. Special Emphasis Notice (SEN): AHRQ Announces Interest in Priority Populations Research (Notice Number: 
NOT-HS-11-014) [Internet]. 2011. Acceso 10 Ago 2011. Hallado en: http://grants.nih.gov/grants/guide/notice-files/NOT-HS-11-014.html

9. Chiesa C. CMR Marketing Relacional: innovando en la actitud de servicio. 1st ed. Providencia, Chile: Eds. Planeta Chilena; 2009. Charpter I; p. 19-77.

10.Castillo E. Escala Multidimensional SERVQUAL [Internet]. 2005. Acceso 23 Ene 2011. Hallado en: http://www.gestiopolis.com/marketing/servqualmedicion-calidad-servicio.pdf

11.MINSAL. Misión y Visión. S.f. Acceso el 03 de Ago 2011. Hallado en $\mathrm{http}: / /$ www.redsalud.gov.cl/portal/url/page/minsalcl/g_conozcanos/g_mision_visi on/presentacion_mision_vision.html

12. Aguilera X, González C, Guerrero A, Bedregal P, Milosavljevic V, Rivera M, Vega J, salinas J, Otaíza F, Espejo F, Espinoza C. Objetivos sanitarios para la década del 2000-2010. El vigía [Internet]. Abril 2002. Acceso el 04 Ago 2011; 5(15):1-12. Hallado

en: http://www.google.cl/url?sa=t\&rct=j\&q=\&esrc=s\&source $=$ web\&cd $=1 \& s q i=2 \&$ ved =0CCkQFjAA\&url=http\%3A\%2F\%2Fepi.minsal.cl\%2Fepi\%2Fhtml\%2Felvigia\% 2Fvigia15.pdf\&ei=CLmdT7WvO4XWtgfZuNSmBA\&usg=AFQjCNFQKEI5N5eT HB8SERaHgpKvmBf58Q\&sig2=zYj10FyjU6O_E3Jk90GfuQ

13.MINSAL. Objetivos Sanitarios para la Década 2000-2010 Evaluación a mitad de periodo [Internet]. S.f. Acceso el 7 de Abril del 2012. Hallado en http://epi.minsal.cl/epi/html/sdesalud/OS/EvOS_IV.pdf

14.Pérez Cárdenas AM. Calidad de servicio como estrategia en la administración pública de los servicios de salud. RIES [Revista en Internet]. 2006. Acceso el 03 Ago 2011; 8(2-A),30-48. Hallado en: http://arimaca.unimagdalena.edu.co/editorial/revistas/index.php/clio_america/art icle/view/29/32

15. Velandia F, Ardón N, Jara M, Cásdenas J, Pérez, N. Oportunidad, satisfacción y razones de no uso de los servicios de salud en Colombia, según la encuesta de calidad de vida del DANE, 1997. Rev Sal Pub [Revista en Internet]. 2004. Acceso el 03 Ago 201; 5(1),46-58. Hallado en: http://www.revistas.unal.edu.co/index.php/revsaludpublica/article/view/18399/19 777

16. Riveros SJ, Berné MC. Análisis de la opinión de usuarios sobre calidad percibida y satisfacción con hospitales públicos: Estudio de caso desde la perspectiva de la aplicación del marketing. Rev méd Chile [Revista en Internet]. 2007 Jul. Acceso el 03 Ago 2011; 135(7): 862-870. Hallado en: http://www.scielo.cl/scielo.php?script=sci_arttext\&pid=S003498872007000700006\&lng=es. doi: 10.4067/S0034-98872007000700006.

17. Al-Mandhari AS, Hassan AA, Haran D. Association between perceived health status and satisfaction with quality of care: evidence from the users of primary health care in Oman. Fam Pract [Revista en Internet]. 2004. Acceso el 03 Ago 2011; 21 (5): 519-527. Hallado en: http://fampra.oxfordjournals.org/content/21/5/519.full.pdf+html

18. Patterson M, Rick J, Wood S, Carroll C, Balain S, Booth A. Systematic review of the links between human resource management practices and performance. Health Technol Assess [Revista en Internet]. 2010 Oct. Acceso el 03 Ago 2011;14(51):1-334, iv. Hallado en: http://www.hta.ac.uk/fullmono/mon1451.pdf

19.Jha AK, Orav EJ, Zheng J, Epstein AM. Patients' perception of hospital care in the United States. N Engl J Med [Revista en Internet]. 2008. Acceso 03 Ago 2011; 359:1921-31. Hallado en: http://www.nejm.org/doi/pdf/10.1056/NEJMsa0804116 
20. Royal College of Nursing. Measuring for quality in health and social care. An RCN position statement [Internet]. 2009. Acceso el 30 de Marzo 2011. Hallado en:

http://www.rcn.org.uk/development/practice/clinical_governance/quality_improv ement/rcn_publications

21. Caminal J. La medida de la satisfacción: un instrumento de participación de la población en la mejora de la calidad de los servicios sanitarios. Rev Cal Asis [Revista en Internet]. 2001. Acceso el 03 Ago 2011;16:276-9. Hallado en: http://www.bvsde.paho.org/bvsacd/cd41/v124.pdf

22. Llanos F, Rosas A, Mendoza D, Contreras C. Comparación de las escalas Likert y Vigesimal para la evaluación de satisfacción de atención en un hospital del Perú. Rev Med Hered [Revista en Internet]. 2001. Acceso el 03 Ago 2011;12(2): 52-57. Hallado en: http://www.scielo.org.pe/pdf/rmh/v12n2/v12n2ao2.pdf

23. Salvador CM. La percepción del cliente de los elementos determinantes de la calidad del servicio universitario: características del servicio y habilidades profesionales. Rev Pap Psic [Revista en Internet]. 2005 ene- abr. Acceso el 03 Ago 2011;26(90), 1-9. Hallado en: http://redalyc.uaemex.mx/redalyc/src/inicio/ArtPdfRed.jsp?iCve=77809001

24. Vicuña M. Nivel de satisfacción y disfunciones percibidas sobre la calidad de atención de los servicios obstétricos. An Fac Med (Perú) [Revista en Internet]. 2002. Acceso el 03 Ago 2011; 63(1):40-50. Hallado en: http://sisbib.unmsm.edu.pe/bvrevistas/anales/v63_n1/pdf/nivel_satisfaccion.pdf

25. Riveros SJ, Berné MC, Múgica JM. Gestión y satisfacción en servicios de salud de Chile: Contraste entre las percepciones de los funcionarios y los usuarios. Rev Med Chil [Revista en Internet]. 2010. Acceso el 03 Ago 2011; 138(5): 630-638. Hallado

http://www.scielo.cl/scielo.php?script=sci_arttext\&pid=S003498872010000500015\&lng=es. doi: 10.4067/S0034-98872010000500015.

26. Garroutte EM, Sarkisian N, Goldberg J, Buchwald D, Beals J. Perceptions of medical interactions between healthcare providers and american Indian older adults. NIH Public Access, Soc Sci Med [Revista en Internet]. 2008 August. Acceso el 03 Ago 2011; 67(4): 546-556. doi:10.1016/j.socscimed.2008.04.015. Hallado en: http://www.ncbi.nlm.nih.gov/pmc/articles/PMC2654200/pdf/nihms66199.pdf

27. Andén A, Andersson SO, Rudebeck CE. Satisfaction is not all--patients' perceptions of outcome of general practice consultations, a qualitative study. BMC Fam Pract [Revista en Internet]. 2005 Oct. Acceso el 03 Ago 2011; 24;6:43. Hallado

en: http://www.ncbi.nlm.nih.gov/pmc/articles/PMC1276792/pdf/1471-2296-6-43.pdf

28.Camgöz-Akdag $\mathrm{H}$, Zineldin $\mathrm{M}$. "Quality of health care and patient satisfaction: An exploratory investigation of the $5 \mathrm{Qs}$ model at Turkey". Clin Govern Int J [serie en Internet]. 2010. Acceso el 03 Ago 2011; 15(2):92-101. Hallado en: http://www.emeraldinsight.com/journals.htm?articleid=1858302\&show=html

29. Riveros SJ, Berné MC. La aplicación del marketing en hospitales públicos desde la perspectiva de los funcionarios: El caso de un hospital del sur de Chile. Rev méd Chile [Revista en Internet]. 2006 Mar. Acceso el 03 Ago 2011; 134(3): 353-360. Hallado en: http://www.scielo.cl/scielo.php?script=sci_arttext\&pid=S003498872006000300014\&lng=es. doi: 10.4067/S0034-98872006000300014.

30.Riveros J, Berné C, García M. Orientación al usuario y satisfacción con el servicio: análisis confirmatorio sobre datos de usuarios de hospital al sur de 
Chile. X Congreso Internacional del CLAD sobre la Reforma del Estado y de la Administración Pública, Santiago, Chile. Documento Libre [Internet]. 2005. Acceso el 19 Mar $2011 . \quad$ Hallado en: http://cdim.esap.edu.co/BancoConocimiento/O/orientacion_al_usuario_y_satisf accion_con_el_servicio/orientacion_al_usuario_y_satisfaccion_con_el_servicio. asp 